\title{
3D-microscopy of prophase nucleus in the meiosis I of wheat-rye amphihaploids
}

\author{
Loginova D.B. ${ }^{1 *}$, Schubert V. ${ }^{2}$, Houben A. ${ }^{2}$, Salina E.A. ${ }^{1}$, Silkova O.G. ${ }^{1}$ \\ ${ }^{1}$ Institute of Cytology and Genetics, SB RAS, Novosibirsk, Russia \\ ${ }^{2}$ Leibniz Institute of Plant Genetics and Crop Plant Research (IPK), Gatersleben, Germany \\ *e-mail:loginova@bionet.nsc.ru
}

In wheat-rye hybrids there is no homologous chromosome pairing. In this regard, hybrids can be used as a model to study disorders of meiotic prophase I. The goal of the present study was to understand the structural and functional organization of prophase I nuclei in amphihaploids with different genetic background (Silkova and Loginova, 2016). Combination of immunostaining with antibodies against ASY1, CENH3, and ZYP1 with confocal and high-resolution microscopy (3D-SIM) enable to understand the centromeres and synaptonemal complex (SC) dynamics and organization. CENH3 signals differed in number, size and shape during prophase I propagation. Differences between bivalent and univalent centromere organization were seen more clearly at pachytene stage when we used 3D-SIM. The dynamic of SC component loading mostly were studied with confocal microscopy, but structure organization at some cases we analyzed with 3D-SIM. SC dynamics at diplotene until diakinesis in wheat is similar to the SC dynamics described for rye, but differs from that of rye during pachytene. In the wheat-rye hybrids, despite the lack of homologues, the loading of ZYP1 occurred. Multiple long extended (linear) signals of ZYP1 appeared at zygotene. Disappearance of anti-ASY1 after full loading of ZYP1 at zygotene and pachytene was observed. As a result, at diakinesis almost all chromosomes are univalent, which indicates desynapsis and the normal functioning of $P h$-locus.

Acknowledgements: This work was supported by RFBR 17-04-01014 and COST STSM CA16212-41305. 